\title{
Countable extreme Gibbs states in a one-dimensional model with unique ground state
}

\author{
Azer Kerimov† \\ Department of Mathematics, Bilkent University, 06533 Bilkent, Ankara, Turkey
}

Received 29 October 1997

Abstract. A one-dimensional model having a unique ground state and countable number of extreme limit Gibbs states is constructed.

\section{The main result}

During recent decades the problem of phase transitions in one-dimensional models has been studied extensively [1-6]. In [7] the following conjecture was formulated: any onedimensional model with discrete (at most countable) spin space and with a unique ground state has a unique Gibbs state if the spin space of this model is finite or the potential of this model is translationally invariant.

The conjecture originates from [7], where it is proved that in a one-dimensional antiferromagnetical model with the Hamiltonian

$$
\sum_{\mathrm{H}(\phi(\mathrm{x}))=x, y \in \mathbf{Z}^{1} ; x>y} U(x-y) \varphi(x) \varphi(y)-\mu \sum_{x \in \mathbf{Z}^{1}} \varphi(x)
$$

where $\mu$ is the external field and the potential $\mathrm{U}(\mathrm{x})$ is non-negative convex function which is extendible to a twice continuously differentiable function such that $\mathrm{U}(\mathrm{x}) \sim \mathrm{Ax}^{-\gamma}, \mathrm{U}^{0} \sim$ $-\mathrm{A} \gamma \mathrm{x}^{-\gamma-1}, \mathrm{U}^{00}\left(\mathrm{x}^{)} \sim \mathrm{A} \gamma(\gamma+1) \mathrm{x}^{-\gamma-2}\right.$ at ${ }^{\mathrm{x}} \rightarrow \infty$; where $\gamma>1$, and $\mathrm{A}$ is a strong positive constant, has a unique ground state at low temperatures.

The ground states of this model are functions of the external field and this relation is very sophisticated $[8,9]$. It turns out that at any fixed value of the external field this model has a unique ground state to within translations. The method of [7] substantially uses the facts that the model is one dimensional, the ground state of the model is unique (to within translations) and that the ground state satisfies the Peierls condition. The uniqueness of the limit Gibbs states is proved by showing that since the ground state is unique the configuration with the minimal energy at any boundary conditions almost coincides with the ground state and the dependence of any event $\phi(\mathrm{A})$ on the boundary conditions can be expressed via the sum of terms connecting A with the boundary, and since the dimension is one (the terms connecting A with the boundary are very long and their entropy is not large enough) this dependence is very weak. 
Since in two- or more dimensional models with a unique ground state and with the Gibbs state related to the ground state different entropy Gibbs states are possible (like those in the Potts model) the conjecture is formulated in one dimension.

† E-mail address: kerimov@fen.bilkent.edu.tr

0305-4470/98/122815+07\$19.50 C 1998 IOP Publishing Ltd

In [7] it is shown that the violation of the conditions of the conjecture leads to the existence of a phase transition in a model with a unique ground state. In this paper we generalize the result of [7] by defining a model (1) (with countable spin space and nontranslationally invariant potential) with a unique ground state having infinitely many extreme limit Gibbs states.

In one dimension any limit Gibbs state of the model is translationally invariant [8]. Moreover, limit Gibbs states of any model satisfying the condition

$$
\mid \sum_{A \subset(-\infty, m] ; B \subset[m, \infty)} U\left(\varphi(B \cup A)-U\left(\varphi(B+l \cup A) \mid<{ }^{\text {constant }}\right.\right.
$$

where the inequality is held uniformly with respect to $\phi(x)$ and integers $m, 1$ are translationally invariant. This fact is a good reason for the absence of phase transitions in antiferromagnetic models. Below we construct a ferromagnetical model with countably many limit Gibbs states.

Consider a ferromagnetical model on $\mathbf{Z}^{1}$ with the Hamiltonian

$$
H(\varphi(x))=\sum_{x \in Z^{1}} U_{x, x+1}^{1}(\varphi(x), \varphi(x+1))+U_{x}^{2}(\varphi(x)) .
$$

The spin space 8 of the model (1) consists of a countable number of alpha spins $\alpha_{i}^{m}$, where $\mathrm{m}, \mathrm{i}=1,2, \ldots$ and a gamma spin $\gamma$. All spins are two-dimensional vectors: $\gamma=(1,0)$ and $\alpha_{i}^{m}$ is a vector $\left(\cos \theta_{\mathrm{m}}, \sin \theta_{\mathrm{m}}\right)$ of ith colour, where $\theta_{\mathrm{m}}=2 \pi\left(1-1 / 2^{\mathrm{m}}\right)$. The

zero-interaction measure $\lambda$ on the space 8 is a counting measure $[10,11]$.

Below we define the functions $U_{x, x+1}^{1}(\varphi(x), \varphi(x+1))$ and $U_{x}^{2}(\varphi(x))$. The first function $\mathrm{U}_{\mathrm{x}, \mathrm{x}}{ }^{1}+1(\phi(\mathrm{x}), \phi(\mathrm{x}+1))$ is bounded in any finite volume and the second functionm it only controls the number of 'admitted' $U_{x}{ }^{2}(\phi(x)) \alpha_{i m}$ plays the role of the external field (at fixed spins).

The pair potential function of nearest neighbours $U_{x, x+1}^{1}(\varphi(x), \varphi(x+1))$ is symmetric with respect to the two arguments

$$
\mathrm{U}_{\mathrm{x}, \mathrm{x}}{ }^{1}+1(\phi 0, \phi 00)=\mathrm{U}_{\mathrm{x}, \mathrm{x}}{ }^{1}+1(\phi 00, \phi 0)
$$

and

$$
U_{x, x+1}^{1}(\varphi(x), \varphi(x+1))=U_{-x-1,-x}^{1}(\varphi(-x-1), \varphi(-x)) .
$$

For non-negative $^{x \in \mathbf{Z}^{1}} U_{x, x+1}^{1}(\varphi(x), \varphi(x+1))_{\text {is defined as }(\mathrm{m}, \mathrm{i}, \mathrm{j}=1,2, \ldots)}$

$$
\begin{array}{cc}
\mathrm{U}_{\mathrm{x}, \mathrm{x} 1+1}(\gamma, \gamma)=0 & \mathrm{U}_{\mathrm{x}, \mathrm{x} 1+1}\left(\alpha_{\mathrm{m}, \gamma}\right)=\mathrm{m} \\
\mathrm{U}_{\mathrm{x}, \mathrm{x} 1+1}\left(\alpha_{\mathrm{i}}, \alpha_{\mathrm{j} m}\right)=\mathrm{m} & \mathrm{U}_{\mathrm{x}, \mathrm{x} 1+1}\left(\alpha_{\mathrm{i}}, \alpha_{\mathrm{j} k}\right)=\mathrm{f}_{\mathrm{x}}(\mathrm{m}, \mathrm{k})
\end{array}
$$

where 


$$
f_{x}(m, k)=-\ln \left(\left(\left(\frac{4}{3}\right)^{1 / 2^{x}}-1\right) / 2\right)+m+k .
$$

The function $U_{x}^{2}(\varphi(x))$ playing the role of the external field is symmetric with respect to the point $x=\frac{1}{2}$. Thus, $U_{x-1}^{2}(\varphi(x-1))=U_{-x}^{2}(\varphi(-x))$ for $x>0$. For positive $\mathrm{x} \in \mathbf{Z}^{1}$

$U_{x}^{2}(\varphi(x))$ is defined as

$U_{x}^{2}(\gamma)=0 \quad U_{x}^{2}\left(\alpha_{i}^{m}\right)=0 \quad$ if $\quad$ i $6 \mathrm{~g}_{\mathrm{x}} \quad U_{x}^{2}\left(\alpha_{i}^{m}\right)=\infty \quad$ if $\quad \mathrm{i}>\mathrm{g}_{\mathrm{x}}$

where

$$
g_{x}=2\left(\left(\frac{4}{3}\right)^{1 / 2^{x}}-1\right)^{-1} .
$$

It can be readily verified that the configuration $\phi(x)=\gamma, x \in Z^{1}$ is the only ground state of the model (1).

The model constructed in [7] has three types of spins: $\alpha, \beta$ and $\gamma$. The unique ground state of the model is a constant configuration $\phi(x)=\gamma$ and the $\alpha$ and $\beta$ spins generating two extreme Gibbs states are symmetric with respect to each other. The model (1) has countable types of spins: $\alpha^{\mathrm{m}}$ and $\gamma$. At each value of the external field the finite number of different $\alpha^{\mathrm{m}}$ spins, namely $\alpha_{i}^{m}$ spins are allowed. But the number of allowed $\alpha_{i}^{m}$ spins at any fixed value of $\mathrm{m}$ is not uniformly bounded: the number of allowed spins tends to infinity as $\mathrm{x}$ grows.

In this model the unique ground state of the model is a constant configuration $\phi(x)=\gamma$ but the $\alpha^{\mathrm{m}}$ spins are not symmetric. Since we have countable types of spins for the convergence of the partition function (lemma 1) we use the conditions (2) and for guaranteeing of lemma 3 we define the function $\mathrm{f}_{\mathrm{x}}(\mathrm{m}, \mathrm{k})$ as a function depending on $\mathrm{x}$ (as in [7]) plus a term involving $\mathrm{m}$ and $\mathrm{k}$.

Let $\mathrm{I}_{\mathrm{V}}$ be the segment $[-\mathrm{V},+\mathrm{V}]$. Suppose the boundary conditions $\phi^{1}(\mathrm{x})=\phi^{1}(\mathrm{x}), \mathrm{x} \in$

$\mathbf{Z}^{1}-\mathrm{IV}_{\mathrm{V}}$ are fixed and

$$
H_{V}\left(\varphi(x) \mid \varphi^{1}(x)\right)=\sum_{x=-V-1}^{V} U_{x, x+1}^{1}(\varphi(x), \varphi(x+1))+\sum_{x=-V}^{V} U_{x}^{2}(\varphi(x)) .
$$

Let us define the partition function

$$
\Xi_{V}=\sum_{\varphi(x) \in \Phi_{V}} \exp \left(-\beta H_{V}\left(\varphi(x) \mid \varphi^{1}(x)\right)\right)
$$

corresponding to the boundary conditions $\phi^{1}(\mathrm{x}), \mathrm{x} \in \mathbf{Z}^{1}-\mathrm{IV}_{\mathrm{V}}$ is finite.

Lemma 1. For any fixed value of $\mathrm{V} 4_{\mathrm{V}}<\infty$.

It follows from lemma 1 that Gibbs distribution in any volume $\mathrm{V}$ corresponding to arbitrary boundary conditions $\phi^{1}(\mathrm{x}), \mathrm{x} \in \mathbf{Z}^{1}-\mathrm{I}_{\mathrm{V}}$ is well defined. In further calculations we restrict the value of the temperature by $\beta>1$, where $\beta^{-1}=\mathrm{kT}, \mathrm{T}$ is the temperature, $\mathrm{k}$ is the Boltzmann constant.

Theorem. Let $\mathrm{T}<1$. For any $\mathrm{m}$ there exists limit Gibbs state of the model (1) $\mathrm{P}^{\alpha_{\mathrm{m}}}$ such that

$$
{ }_{\mathrm{P}}^{\alpha^{m}}\left(\varphi(0)=\alpha^{m}\right)=\sum_{i=1}^{\infty} \boldsymbol{P}^{\alpha^{m}}\left(\varphi(0)=\alpha_{i}^{m}\right)>\frac{1}{2} \text {. }
$$




\section{Proofs}

We start this section with the proof of lemma 1.

Proof of lemma 1. Let us fix $\mathrm{V}$ and the boundary conditions $\phi^{1}(\mathrm{x}), \mathrm{x} \in \mathbf{Z}^{1}-\mathrm{IV}_{\mathrm{V}}$ :

$$
\begin{aligned}
\Xi_{V} & =\sum_{\varphi(x) \in \Phi_{V}} \exp \left(-\beta H_{V}\left(\varphi(x) \mid \varphi^{1}(x)\right)\right) \\
& =\sum_{\varphi(x) \in \Phi_{V}} \exp \left(-\beta \sum_{x=-V-1}^{V} U_{x, x+1}^{1}(\varphi(x), \varphi(x+1))+\sum_{x=-V}^{V} U_{x}^{2}(\varphi(x))\right) \\
& =\sum_{\varphi(x) \in \Phi_{V}} \prod_{x=-V-1}^{V} \exp \left(-\beta U_{x, x+1}^{1}(\varphi(x), \varphi(x+1))\right) \prod_{x=-V}^{V} \exp \left(-\beta U_{x}^{2}(\varphi(x))\right) .
\end{aligned}
$$

The notation $\gamma=\alpha_{0}^{0}$ will be convenient for further calculations.

The last

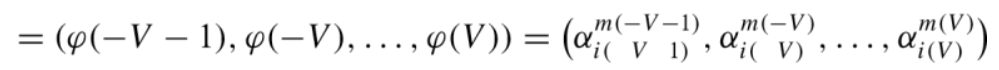

summation is taken over all possible configurations,

$\phi(\mathrm{x})$

where $\mathrm{m}$ and $\mathrm{i}$ take all non-negative integers, and the indices of $\alpha$ are both together zeros or non-zeros.

Thus, the partition function can be written as

$$
\begin{aligned}
\Xi_{V}= & \sum_{(m(-V-1), m(-V), \ldots, m(V))} \sum_{(\mathrm{i}(-V-1), \mathrm{i}(-V), \ldots, \mathrm{i}(V))} \\
& \times \prod_{x=-V-1}^{V} \exp \left(-\beta U_{x, x+1}^{1}(\varphi(x), \varphi(x+1))\right) \prod_{x=-V}^{V} \exp \left(-\beta U_{x}^{2}(\varphi(x))\right) .
\end{aligned}
$$

$$
\begin{array}{rlr}
\Xi_{V} & =\sum_{1} \prod_{x=-V-1}^{V} \exp \left(-\beta U_{x, x+1}^{1}(\varphi(x), \varphi(x+1))\right) \prod_{x=-V}^{V} \exp \left(-\beta U_{x}^{2}(\varphi(x))\right) & \begin{array}{r}
\text { Due to (3) } \\
\text { for each fixed } \\
\text { collection }
\end{array} \\
& =\sum_{1} \prod_{x=-V-1}^{V} \exp \left(-\beta U_{x, x+1}^{1}(\varphi(x), \varphi(x+1))\right)<\infty & \left(\mathrm{m}^{0}(-\mathrm{V}-\right.
\end{array}
$$
$\left.1), \mathrm{m}^{0}(-\mathrm{V}), \ldots, \mathrm{m}^{0}(\mathrm{~V})\right)$ there are just a finite number of collections $(\mathrm{i}(-\mathrm{V}-1), \mathrm{i}(\mathrm{V}), \ldots, \mathrm{i}(\mathrm{V}))$, such that the corresponding term in the summation (5) is non-zero (for others $\mathrm{U}_{\mathrm{x}^{2}}(\phi(\mathrm{x}))=\infty$ ).

Therefore, in order to prove the lemma, it is enough to show that where in $\mathrm{P}_{1}$ the summation is taken over all possible configurations

$$
\left(\alpha_{1}^{m(-V-1)} \text { or } \gamma, \alpha_{1}^{m(-V)} \text { or } \gamma, \ldots, \alpha_{1}^{m(V)} \text { or } \gamma\right) .
$$

Now we note that

where

$$
\sum_{1} \prod_{x=-V-1}^{V} \exp \left(-\beta U_{x, x+1}^{1}(\varphi(x), \varphi(x+1))\right) \leqslant \prod_{x=-V-1}^{V} M_{x}
$$

$M_{x}=\sum_{k, l} \exp \left(-\beta U_{x, x+1}^{1}\left(\alpha_{1}^{k}, \alpha_{1}^{l}\right)\right)+\sum_{k} \exp \left(-\beta U_{x, x+1}^{1}\left(\alpha^{k}, \gamma\right)\right)+\exp \left(-\beta U_{x, x+1}^{1}(\gamma, \gamma)\right)$ 
and the summation is taken over all possible natural numbers $\mathrm{k}$ and $\mathrm{l}$. It can be easily shown that due to the conditions (2) $\mathrm{M}_{\mathrm{x}}$ is finite. The lemma is proved.

Define the Gibbs distribution $\mathrm{P}_{\mathrm{V}}\left(\phi(\mathrm{x}) \mid \phi^{\alpha_{\mathrm{m}}}\right)$ in the space ${ }_{m} \Phi_{V}$ corresponding to the boundary conditions $\varphi^{\alpha}(x)=\alpha_{1}^{m}, x \in \mathbf{Z}^{1}-\left[-V, V_{m}\right]$. Let $\mathrm{P}^{\alpha}$ be a limit point of the sequence of Gibbs distributions $\mathrm{P}_{\mathrm{V}}\left(\phi(\mathrm{x}) \mid \phi^{\alpha}\right)$ when $\mathrm{V}$ goes to infinity. This limit point $\mathrm{P}{ }_{\mathrm{m}}$ is a limit Gibbs state of the model (1) $[8,9]$.

Proof of the theorem. In order to prove the theorem we show that $\mathrm{P}^{\alpha_{\mathrm{m}}}$ satisfies (4).

To prove (4) it is enough to show that at any value of $V \boldsymbol{P}_{V}\left(\varphi(0)=\alpha^{m} \mid \varphi^{\alpha^{m}}\right) \geqslant \frac{9}{16}$.

Let $\mathrm{P} V\left(\varphi(x)=\alpha^{m}, x \in[-V, V] \mid \varphi^{\alpha^{m}}\right)=\boldsymbol{P}_{V}\left(\bigcap_{x=-V}^{V} \bigcup_{i=1}^{\infty}\left(\varphi(x)=\alpha_{i}^{m} \mid \varphi^{\alpha^{m}}\right)\right.$. Obviously, $\mathrm{P}_{\mathrm{V}}\left(\phi(0)=\alpha^{\mathrm{m}} \mid \phi^{\alpha_{\mathrm{m}}}\right)>\mathrm{P}_{\mathrm{V}}\left(\phi(\mathrm{x})=\alpha^{\mathrm{m}}, \mathrm{x} \in[-\mathrm{V}, \mathrm{V}] \mid \phi^{\alpha_{\mathrm{m}}}\right)$. In order to

prove (4) we shall prove that

$$
\mathrm{P}^{V}\left(\varphi(x)=\alpha^{m}, x \in[-V, V] \mid \varphi^{\alpha^{m}}\right) \geqslant \frac{9}{16}
$$

Define a Gibbs

$$
\varphi^{\alpha, l e f t}(x)=\alpha_{1}^{m}, x \in\left(-\infty,-V-\text { distribution } \mathrm{P}_{\mathrm{V}}\left(\phi(\mathrm{x}) \mid \phi^{\alpha_{\mathrm{m}}, \text { left }}\right)\right. \text { in }
$$

the space $8_{\mathrm{V}}$ corresponding to the $\mathrm{m}_{\mathrm{m}}$ boundary conditions $\left.\mathrm{m}_{\mathrm{m}} 1\right]$ and $\phi^{\alpha, \mathrm{left}}(\mathrm{x})=\emptyset, \mathrm{x} \in$

$[\mathrm{V}+1, \infty)$.

By definition

$$
\begin{aligned}
& { }_{V}\left(\varphi(x)=\alpha^{m} \mid \varphi^{\alpha^{m}}\right)=\frac{\sum_{\alpha^{m}} \exp \left(-\beta\left(H\left(\varphi(x) \mid \varphi^{\alpha^{m}}\right)\right)\right)}{\sum \exp \left(-\beta\left(H\left(\varphi(x) \mid \varphi^{\alpha^{m}}\right)\right)\right)} \\
& \mathrm{P} \quad(\quad( \\
& { }_{\mathrm{P}}\left(\varphi(x)=\alpha^{m} \mid \varphi^{\alpha^{m}, \text { left }}\right)=\frac{\sum_{\alpha^{m}} \exp \left(-\beta\left(H\left(\varphi(x) \mid \varphi^{\alpha^{m}, \text { left }}\right)\right)\right)}{\sum \exp \left(-\beta\left(H\left(\varphi(x) \mid \varphi^{\alpha^{m}, \text { left }}\right)\right)\right)}
\end{aligned}
$$

where the summations in both numerators are taken over all configurations $\phi(\mathrm{x}) \in 8_{\mathrm{V}}$ such that $=\alpha_{i}^{m} \phi(\mathrm{x})$ for some $\mathrm{i}$ and both summations in the denominators are taken over all configurations $\phi(\mathrm{x}) \in 8 \mathrm{v}$.

In the model (1) 'adjacent' spins ( $\alpha_{i}^{m}$ spins at fixed $\mathrm{m}$ or $\gamma$ spins) tend to be aligned. That is, the Hamiltonian (1) can be interpreted as ferromagnetic and the following inequality is a natural ferromagnetic inequality.

Lemma 2.

$$
\mathrm{P}_{\mathrm{V}}\left(\phi(\mathrm{x})=\alpha^{\mathrm{m}}, \mathrm{x} \in[-\mathrm{V}, \mathrm{V}] \mid \phi^{\alpha_{\mathrm{m}}, \mathrm{left}}\right) 6 \mathrm{P}_{\mathrm{V}}\left(\phi(\mathrm{x})=\alpha^{\mathrm{m}}, \mathrm{x} \in[-\mathrm{V}, \mathrm{V}] \mid \phi^{\alpha_{\mathrm{m}}}\right)
$$

Proof. Let us compare numerators and denominators of (7) and (8). Each term in the numerator of $(7)$ is equal to $\exp (-\mathrm{m} \beta)$ times the corresponding term of $(8)$. Each term in the denominator of $(7)$ is equal to $\exp (-\mathrm{m} \beta)$ (respectively $\left.\exp \left(-\mathrm{f}_{\mathrm{x}} \beta\right)\right)$ times the corresponding term of $(8)$ if at $\mathrm{x}=\mathrm{V} \phi(\mathrm{x})=\alpha_{\mathrm{i}}^{\mathrm{m}}$ for some $\mathrm{i}$ or $\phi(\mathrm{x})=\gamma\left(\right.$ respectively $\phi(\mathrm{x})=\alpha_{\mathrm{j}}^{\mathrm{k}}$ for some $\mathrm{k} 6=$ $\mathrm{m}$ and $\mathrm{j})$.

Since $f_{x}>m$, the lemma is proved. 
It follows from lemma 2 that the theorem is a consequence of the following.

Lemma 3.

$$
\mathrm{P} V\left(\varphi(x)=\alpha^{m}, x \in[-V, V] \mid \varphi^{\alpha^{m}, l e f t}\right) \geqslant \frac{9}{16} .
$$

Proof. Consider a Markov chain (non-homogeneous) starting at point $\mathrm{x}=-\mathrm{V}$ and ending at point $\mathrm{x}=\mathrm{V}$ with initial condition $\phi(-\mathrm{V}-1)=\alpha^{1}$ with transition probabilities $\pi_{\xi(\mathrm{x}), \xi(\mathrm{x}+1)}$ $\left(\pi_{\xi(x), \xi(x+1)}\right.$ is the probability of the event that $\phi(x+1)=\xi(x+1)$ under the condition that $\phi(x)$ $=\xi(\mathrm{x}))$, where

$$
\pi_{\xi(\mathrm{x}), \xi(\mathrm{x}+1)}=\mathrm{P}_{\mathrm{V}}(\phi(\mathrm{x}+1)=\xi(\mathrm{x}+1) \mid \phi(\mathrm{x})=\xi(\mathrm{x}), \phi(\mathrm{x}+2)=\emptyset)
$$

It follows from the definitions that

$$
v\left(\varphi(x)=\xi(x), x \in[-V, V] \mid \varphi^{\alpha^{m}, l e f t}\right)=\pi_{\xi(-V-1)=\alpha_{1}^{m}, \xi(-V)} \prod_{x}^{-} \pi_{V(x), \xi(x+1)} \quad \mathrm{P} . \quad 1
$$

Define $\pi_{\xi}^{\xi}(\mathrm{x})=\alpha_{\mathrm{m}}, \xi(\mathrm{x}+1)=\alpha_{\mathrm{m}}=\mathrm{P} \pi \xi(\mathrm{x})=\alpha_{\mathrm{im}}, \xi(\mathrm{x}+1)=\alpha_{\mathrm{j} m}$, where the summation is taken over all possible values of $\mathrm{j}$ (by definition the sum consists of a finite number of terms (due to (3)) and does not depend on i).

Thus,

$$
\begin{aligned}
& { }_{V}\left(\varphi(x)=\alpha^{m}, x \in[-V, V] \mid \varphi^{\alpha^{m}, \text { left }}\right)=\pi_{\xi(-V-1)=\alpha_{1}^{m}, \xi(-V)=\alpha^{m}} \prod_{x}^{-} \pi_{V} \pi_{\xi(x)=\alpha^{m}, \xi(x+1)=\alpha^{m}} \quad \begin{array}{l}
1 \\
\mathrm{P}
\end{array} \\
& =\prod_{x=-V-1}^{V-1} \pi_{\xi(x)=\alpha^{m}, \xi(x+1)=\alpha^{m}} .
\end{aligned}
$$

Due to definitions $(\beta>1)$,

$\pi_{\xi(x)=\alpha^{m}, \xi(x+1)=\alpha^{m}}$

$$
\begin{aligned}
& =\frac{\sum_{i=1}^{g(x+1)} \exp (-\beta m)}{\sum_{i=1}^{g(x+1)} \exp (-\beta m)+\sum_{k=1, k \neq m}^{\infty} \sum_{i=1}^{g(x+1)} \exp \left(-\beta f_{x+1}(m, k)\right)+\exp (-\beta m)} \\
& =\frac{g(x+1) \exp (-\beta m)}{g(x+1) \exp (-\beta m)+\sum_{k=1, k \neq m}^{\infty} g(x+1) \exp \left(-\beta f_{x+1}(m, k)\right)+\exp (-\beta m)} \\
& =\frac{1}{1+\sum_{k=1, k \neq m}^{\infty} \exp \left(-\beta\left(f_{x+1}(m, k)-m\right)\right)+1 / g(x+1)} \\
& \geqslant \frac{1}{1+\sum_{k=1, k \neq m}^{\infty} \exp \left(-f_{x+1}(m, k)+m\right)+1 / g(x+1)} \\
& \geqslant \frac{1}{1+\exp \left(-f_{x+1}(m, k)+m+k\right)+1 / g(x+1)} \geqslant\left(\frac{3}{4}\right)^{1 / 2^{x+1}} .
\end{aligned}
$$

Since 


$$
{ }_{V}\left(\varphi(x)=\alpha^{m}, x \in[-V, V] \mid \varphi^{\alpha^{m}, \text { left }}\right) \geqslant\left(\prod_{x}^{\infty} \pi_{\xi(x)=\alpha^{m}, \xi(x+1)=\alpha^{m}}\right)^{\mathrm{P}}=
$$

in order to prove (9) it is enough to show that

$$
\prod_{x=0}^{\infty} \pi_{\xi(x)=\alpha^{m}, \xi(x+1)=\alpha^{m}} \geqslant \frac{3}{4} \text {. }
$$

The last inequality (10) follows directly:

$$
\prod_{x=0}^{\infty} \pi_{\xi(x)=\alpha^{m}, \xi(x+1)=\alpha^{m}} \geqslant \prod_{x=1}^{\infty}\left(\frac{3}{4}\right)^{1 / 2^{x}}=\frac{3}{4} .
$$

The inequality (10), and hence lemma 3 is proved.

Now the inequality (6) is a direct implication of lemmas 2 and 3 . The theorem follows from the inequality (6).

\section{Final remarks}

The model (1) has the following unusual property:

$$
\mathrm{P}^{\alpha^{m}}\left(\varphi\left(x_{1}\right)=\alpha^{m}, \varphi\left(x_{2}\right)=\alpha^{m}, \ldots, \varphi\left(x_{l}\right)=\alpha^{m}\right)>\frac{1}{2}
$$

where the last inequality is held uniformly with respect to 1 and $\mathrm{x}_{1}, \mathrm{x}_{2}, \ldots, \mathrm{x}_{1}$. The model

(1) also has the following physical meaning.

(i) At zero temperature all spins of the model aligned: $\phi(x)=\gamma$.

(ii) At non-zero temperatures since the ferromagnetical model has a very strong couplingpotential between aligned spins $\alpha_{i}^{m}$ there exist $\alpha^{\mathrm{m}}$ states at which almost all spins are aligned.

\section{Acknowledgment}

The author thanks the referees for their helpful suggestions.

\section{References}

[1] Dyson F J 1969 Commun. Math. Phys. 12 91-107

[2] Spitzer F 1975 J. Func. Anal. 20 240-55

[3] Kalikow S 1977 Ann. Probab. 5 467-9

[4] Sullivan W G 1975 Commun. Dublin Institute for Advanced Studies

[5] Miyamoto M 1984 J. Math. Kyoto University 24 679-88

[6] Kerimov A A 1993 J. Stat. Phys. 72 571-620

[7] Kerimov A A 1996 Physica 225A 271-6

[8] Burkov S E and Sinai Ya G 1983 Usp. Mat. Nauk 38 205-25

[9] Kerimov A A 1984 Theor. Mat. Fiz. 58 473-80

[10] Georgii H O 1988 Gibbs Measures and Phase Transitions (Berlin: De Gruyter)

[11] Sinai Ya G 1982 Theory of Phase Transitions. Rigorous Results (Budapest: Academy of Kiado) 